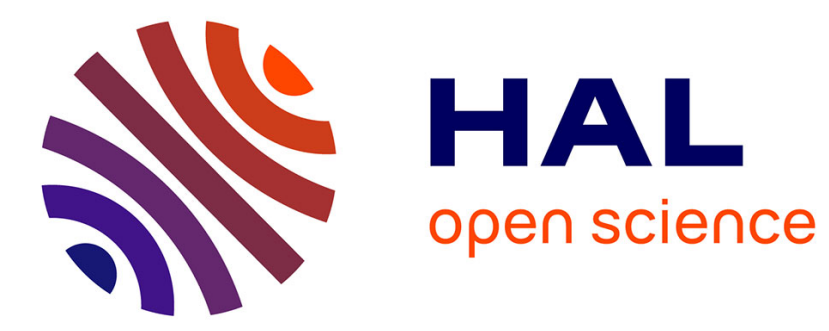

\title{
Edge stress intensity functions in 3-D anisotropic composites
}

Zohar Yosibash, Netta Omer, Monique Dauge

\section{To cite this version:}

Zohar Yosibash, Netta Omer, Monique Dauge. Edge stress intensity functions in 3-D anisotropic composites. Composites Science and Technology, 2008, 68 (5), pp.1216-1224. 10.1016/j.compscitech.2007.07.018 . hal-00352762

HAL Id: hal-00352762

https://hal.science/hal-00352762

Submitted on 11 Dec 2017

HAL is a multi-disciplinary open access archive for the deposit and dissemination of scientific research documents, whether they are published or not. The documents may come from teaching and research institutions in France or abroad, or from public or private research centers.
L'archive ouverte pluridisciplinaire HAL, est destinée au dépôt et à la diffusion de documents scientifiques de niveau recherche, publiés ou non, émanant des établissements d'enseignement et de recherche français ou étrangers, des laboratoires publics ou privés. 


\title{
Edge stress intensity functions in 3-D anisotropic composites
}

\author{
Zohar Yosibash $^{\mathrm{a}, *}$, Netta Omer ${ }^{\mathrm{a}}$, Monique Dauge ${ }^{\mathrm{b}}$ \\ a Pearlstone Center for Aeronautical Studies, Department of Mechanical Engineering, Ben-Gurion University of the Negev, Beer-Sheva 84105, Israel \\ ${ }^{\mathrm{b}}$ UMR CNRS 6625 - IRMAR, Université de Rennes 1 - Campus de Beaulieu, 35042 Rennes cedex, France
}

\begin{abstract}
The elastic solution in the vicinity of a traction free edge and an interface crack in cross-ply anisotropic laminates is provided in details for three problems. Complex eigen-pairs and edge stress intensity functions are computed with high accuracy, aimed to serve as benchmark problems against which other numerical methods can be checked.
\end{abstract}

Keywords: A. Laminate; B. Delamination; C. Finite-element analysis (FEA), Crack; Edge stress intensity factors

\section{Introduction}

Delamination starting at a traction free edge or interface cracks between plies lead most frequently to catastrophic failures in cross-ply laminates. Many of these laminates are made of brittle anisotropic materials and occupy three-dimensional (3-D) domains, therefore the 3-D elastic solution in the vicinity of these edges (a crack front is a particular case of a general edge) is of major engineering interest. The mathematical complexity of the 3-D anisotropic elasticity system in the vicinity of an edge makes it intractable by analytical methods and very complex even for numerical treatment. Therefore most of past studies reduced the problem to two-dimensions addressing the solution in the vicinity of singular points, see, e.g. $[4,6]$. Only very limited studies considered restricted classes of 3-D edge singularity problems, as [2] for instance which investigates the specific through interface crack between a $\pm 45^{\circ}$ transversely isotropic pair of materials.

Recently, the explicit mathematical representation of the 3-D elastic solution in the vicinity of a straight edge has been provided and the quasi-dual function method was

\footnotetext{
${ }^{*}$ Corresponding author.

E-mail addresses: zohary@bgu.ac.il (Z. Yosibash),nettao@bgu.ac.il (N. Omer), Monique.Dauge@univ-rennes1.fr (M. Dauge).
}

proposed for the extraction of edge stress intensity function (ESIF) [1]. This enabled the development of highly accurate numerical methods $[8,7,3]$ for the computation of elastic solutions for three benchmark problems including the full expansion in the vicinity of a traction free edge and interface crack. The problems are suggested to be used as benchmarks against which methods for the computation of edge stress intensity functions can be compared.

\section{The elastic solution in the vicinity of an edge}

Consider a 3-D domain in which a straight edge is of interest (see Fig. 1 for example). A cylindrical coordinate system $\left(r, \theta, x_{3}\right)$ is located at a point on the edge so that the $x_{3}$ axis is along the edge. The two flat planes that intersect at the edge are denoted by $\Gamma_{1}$ and $\Gamma_{2}$. The displacement vector and strain and stress tensors are represented in cylindrical coordinates: $\boldsymbol{u}=\left\{u_{r}, u_{\theta}, u_{x_{3}}\right\}^{\mathrm{T}}, \boldsymbol{\varepsilon}=\left\{\varepsilon_{r r}, \varepsilon_{\theta \theta}\right.$, $\left.\varepsilon_{33}, \gamma_{\theta 3}, \gamma_{r 3}, \gamma_{r \theta}\right\}^{\mathrm{T}}$ and $\boldsymbol{\sigma}=\left\{\sigma_{r r}, \sigma_{\theta \theta}, \sigma_{33}, \sigma_{\theta 3}, \sigma_{r 3}, \sigma_{r \theta}\right\}^{\mathrm{T}}$. The linear elasticity problem is being solved by means of separation of variables, yielding in an elastic solution that can be presented in the vicinity of an edge by the series $[1,8]$ :

$$
\boldsymbol{u}=\sum_{i \geqslant 1} \sum_{j \geqslant 0} \partial_{3}^{j} A_{i}\left(x_{3}\right) \boldsymbol{\Phi}_{j}^{\left(\alpha_{i}\right)}(r, \theta), \quad \boldsymbol{\Phi}_{j}^{\left(\alpha_{i}\right)}(r, \theta)=r^{\alpha_{i}+j} \boldsymbol{\varphi}_{j}^{\left(\alpha_{i}\right)}(\theta)
$$




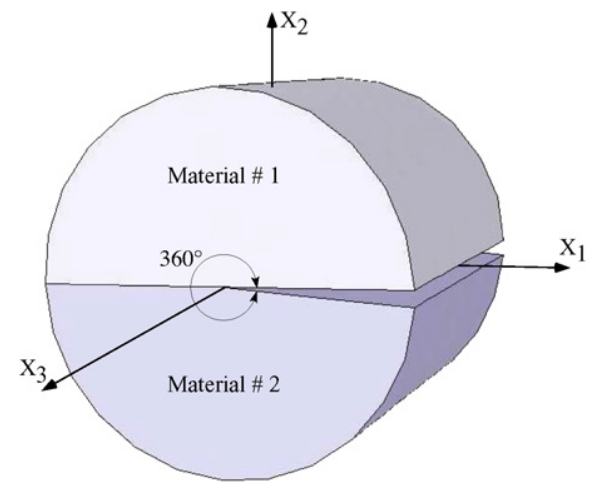

Fig. 1. Bi-material cracked domain.

or in a more explicit form:

$$
\begin{aligned}
\boldsymbol{u}= & A_{1}\left(x_{3}\right) r^{\alpha_{1}} \boldsymbol{\varphi}_{0}^{\left(\alpha_{1}\right)}(\theta)+\partial_{3} A_{1}\left(x_{3}\right) r^{\alpha_{1}+1} \boldsymbol{\varphi}_{1}^{\left(\alpha_{1}\right)}(\theta) \\
& +\partial_{3}^{2} A_{1}\left(x_{3}\right) r^{\alpha_{1}+2} \boldsymbol{\varphi}_{2}^{\left(\alpha_{1}\right)}(\theta)+\cdots \\
& +A_{2}\left(x_{3}\right) r^{\alpha_{2}} \boldsymbol{\varphi}_{0}^{\left(\alpha_{2}\right)}(\theta)+\partial_{3} A_{2}\left(x_{3}\right) r^{\alpha_{2}+1} \boldsymbol{\varphi}_{1}^{\left(\alpha_{2}\right)}(\theta) \\
& +\partial_{3}^{2} A_{2}\left(x_{3}\right) r^{\alpha_{2}+2} \boldsymbol{\varphi}_{2}^{\left(\alpha_{2}\right)}(\theta)+\cdots \\
& +A_{3}\left(x_{3}\right) r^{\alpha_{3}} \boldsymbol{\varphi}_{0}^{\left(\alpha_{3}\right)}(\theta)+\partial_{3} A_{3}\left(x_{3}\right) r^{\alpha_{3}+1} \boldsymbol{\varphi}_{1}^{\left(\alpha_{3}\right)}(\theta) \\
& +\partial_{3}^{2} A_{3}\left(x_{3}\right) r^{\alpha_{3}+2} \boldsymbol{\varphi}_{2}^{\left(\alpha_{3}\right)}(\theta)+\cdots \\
& +\cdots
\end{aligned}
$$

where $A_{i}\left(x_{3}\right)$ is the Edge Stress Intensity Function (ESIF) associated with the $i$ th eigen-pair, the singular function $\boldsymbol{\Phi}_{0}^{\left(\alpha_{i}\right)}=r^{\alpha_{i}} \boldsymbol{\varphi}_{0}^{\left(\alpha_{i}\right)}(\theta)$ being the well known two-dimensional eigen-function, whereas $\boldsymbol{\Phi}_{j}^{\left(\alpha_{i}\right)}=r^{\alpha_{i}+j} \boldsymbol{\varphi}_{j}^{\left(\alpha_{i}\right)}(\theta), j \geqslant 1$ are the shadow functions of the primal singular function. The angular part of eigen-functions and their shadows have three components denoted by:

$\boldsymbol{\varphi}_{j}^{\left(\alpha_{i}\right)}(\theta)=\left(\varphi_{j r}^{\left(\alpha_{i}\right)}(\theta), \varphi_{j \theta}^{\left(\alpha_{i}\right)}(\theta), \varphi_{j x_{3}}^{\left(\alpha_{i}\right)}(\theta)\right)^{\mathrm{T}}, \quad j=0,1, \ldots$

Herein any eigen-function $\left(\varphi_{j r}^{\left(\alpha_{i}\right)}(\theta), \varphi_{j \theta}^{\left(\alpha_{i}\right)}(\theta), \varphi_{j x_{3}}^{\left(\alpha_{i}\right)}(\theta)\right)^{T}$ or any shadow function are expressed by sin, $\cos$ series. For example, if $\varphi_{j r}^{\left(\alpha_{i}\right)}(\theta)$ is to be evaluated then

$$
\begin{aligned}
\varphi_{j r}^{\left(\alpha_{i}\right)}= & C_{1} \sin \left(\alpha_{i} \theta\right)+C_{2} \sin \left(\left(\alpha_{i}+1\right) \theta\right)+C_{3} \sin \left(\left(\alpha_{i}-1\right) \theta\right) \\
& +C_{4} \sin \left(\left(\alpha_{i}+2\right) \theta\right)+C_{5} \sin \left(\left(\alpha_{i}-2\right) \theta\right)+C_{6} \sin \left(\left(\alpha_{i}+3\right) \theta\right) \\
& +C_{7} \sin \left(\left(\alpha_{i}-3\right) \theta\right)+C_{8} \cos \left(\alpha_{i} \theta\right)+C_{9} \cos \left(\left(\alpha_{i}+1\right) \theta\right) \\
& +C_{10} \cos \left(\left(\alpha_{i}-1\right) \theta\right)+C_{11} \cos \left(\left(\alpha_{i}+2\right) \theta\right) \\
& +C_{12} \cos \left(\left(\alpha_{i}-2\right) \theta\right)+C_{13} \cos \left(\left(\alpha_{i}+3\right) \theta\right) \\
& +C_{14} \cos \left(\left(\alpha_{i}-3\right) \theta\right)
\end{aligned}
$$

Remark 1. For traction free boundary conditions in the vicinity of an edge, the first three non-negative eigen-values are zero. These zero eigen-values correspond to three rigid body displacements in the $x_{1}, x_{2}, x_{3}$ directions having constant eigen-vectors and zero stress tensor. The shadows are associated therfore with integers $(\alpha+i=i)$, resulting in polynomials in $x_{1}, x_{2}$ thus do not contribute to singularities but only to the "Taylor-expansion" of the regular part of the solution.

In general, primal and shadows associated with nonnegative integers are polynomials, which do not contribute to the singularities and are of limited engineering interest.
For these, instead of (3) a polynomial expression in $x_{1}, x_{2}$ is more appropriate for the representation of displacements in $x_{1}, x_{2}$ directions.

Remark 2. We have determined that 14 terms in (3) are sufficient to represent accurately the eigen-functions and shadows for the three example problems in Section 3. All data presented herein have a $L^{2}$ relative error of less than $10^{-3} \%$ when (3) with 14 terms is compared to the eigenpairs and shadows computed by very high order finite-element methods documented in [3].

There are cases for which an eigen-value is complex, i.e. $\alpha_{i}=\alpha_{i \Re}+l \alpha_{i \Im}$ and so are its corresponding eigen-function and shadows $\boldsymbol{\varphi}_{j}^{\left(\alpha_{i}\right)}(\theta)=\boldsymbol{\varphi}_{j \Re}^{\left(\alpha_{i}\right)}(\theta)+i \boldsymbol{\varphi}_{j \mathfrak{\mathfrak { S }}}^{\left(\alpha_{i}\right)}(\theta)$, therefore the corresponding ESIF is complex also, $A_{i}\left(x_{3}\right)=A_{i \Re}\left(x_{3}\right)+$ $\imath A_{i \mathfrak{I}}\left(x_{3}\right)$. In this case $\boldsymbol{\varphi}_{j \mathfrak{R}}^{\left(\alpha_{i}\right)}(\theta), \boldsymbol{\varphi}_{j \mathfrak{\Im}}^{\left(\alpha_{i}\right)}(\theta)$ are obtained by substituting $\alpha$ with $\alpha_{i \Re}$ and $\alpha_{i \Im}$ in (3) respectively.

For complex eigen-pairs, $\alpha_{i+1}=\alpha_{i \Re}-\imath \alpha_{i \mathfrak{3}}, \varphi_{j}^{\left(\alpha_{i+1}\right)}(\theta)=$ $\boldsymbol{\varphi}_{j \mathfrak{R}}^{\left(\alpha_{i}\right)}(\theta)-\imath \boldsymbol{\varphi}_{j \mathfrak{\mathcal { Y }}}^{\left(\alpha_{i}\right)}(\theta)$ and $A_{i+1}\left(x_{3}\right)=A_{i \Re}\left(x_{3}\right)-\imath A_{i \mathfrak{\Im}}\left(x_{3}\right)$. For a crack in a bi-material interface for example, the first eigen-value is complex $\alpha_{1, \Re} \pm l \alpha_{1 \mathfrak{I}}$, so that (2) is:

$$
\begin{aligned}
& \boldsymbol{u}=\left(A_{1 \Re}\left(x_{3}\right)+\imath A_{1 \mathfrak{I}}\left(x_{3}\right)\right) r^{\alpha_{1 \Re}+l \alpha_{1 \mathfrak{I}}}\left(\boldsymbol{\varphi}_{0 \Re}^{\left(\alpha_{1}\right)}(\theta)+\imath \boldsymbol{\varphi}_{0 \mathfrak{I}}^{\left(\alpha_{1}\right)}(\theta)\right) \\
& +\left(\partial_{3} A_{1 \Re \mathcal{R}}\left(x_{3}\right)+l \partial_{3} A_{1 \mathfrak{J}}\left(x_{3}\right)\right) r^{1+\alpha_{1 \mathfrak{R}}+l \alpha_{1 \mathfrak{S}}}\left(\boldsymbol{\varphi}_{1 \mathfrak{R}}^{\left(\alpha_{1}\right)}(\theta)\right. \\
& \left.+\imath \boldsymbol{\varphi}_{1 \mathfrak{I}}^{\left(\alpha_{1}\right)}(\theta)\right)+\left(\partial_{3}^{2} A_{1 \Re}\left(x_{3}\right)\right. \\
& \left.+\imath \partial_{3}^{2} A_{1 \mathfrak{I}}\left(x_{3}\right)\right) r^{2+\alpha_{1 \Re}+l \alpha_{1 \mathfrak{I}}}\left(\boldsymbol{\varphi}_{2 \Re}^{\left(\alpha_{1}\right)}(\theta)+\imath \boldsymbol{\varphi}_{2 \mathfrak{J}}^{\left(\alpha_{1}\right)}(\theta)\right)+\cdots \\
& +\left(A_{1 \Re \mathfrak{R}}\left(x_{3}\right)-\imath A_{1 \mathfrak{I}}\left(x_{3}\right)\right) r^{\alpha_{1 \Re}-l \alpha_{1 \mathfrak{I}}}\left(\boldsymbol{\varphi}_{0 \Re}^{\left(\alpha_{1}\right)}(\theta)-\imath \boldsymbol{\varphi}_{0 \mathfrak{I}}^{\left(\alpha_{1}\right)}(\theta)\right) \\
& +\left(\partial_{3} A_{1 \Re}\left(x_{3}\right)-l \partial_{3} A_{1 \mathfrak{I}}\left(x_{3}\right)\right) r^{1+\alpha_{1 \Re}-l \alpha_{1 \mathfrak{S}}}\left(\boldsymbol{\varphi}_{1 \Re}^{\left(\alpha_{1}\right)}(\theta)\right. \\
& \left.-\imath \boldsymbol{\varphi}_{1 \mathfrak{I}}^{\left(\alpha_{1}\right)}(\theta)\right)+\left(\partial_{3}^{2} A_{1 \Re}\left(x_{3}\right)\right. \\
& \left.-\imath \partial_{3}^{2} A_{1 \mathfrak{I}}\left(x_{3}\right)\right) r^{2+\alpha_{1 \Re}-l \alpha_{1 \mathfrak{I}}}\left(\boldsymbol{\varphi}_{2 \Re}^{\left(\alpha_{1}\right)}(\theta)-\imath \boldsymbol{\varphi}_{2 \mathfrak{J}}^{\left(\alpha_{1}\right)}(\theta)\right)+\cdots \\
& +A_{3}\left(x_{3}\right) r^{\alpha_{3}} \boldsymbol{\varphi}_{0}^{\left(\alpha_{3}\right)}(\theta)+\partial_{3} A_{3}\left(x_{3}\right) r^{\alpha_{3}+1} \boldsymbol{\varphi}_{1}^{\left(\alpha_{3}\right)}(\theta) \\
& +\partial_{3}^{2} A_{3}\left(x_{3}\right) r^{\alpha_{3}+2} \varphi_{2}^{\left(\alpha_{3}\right)}(\theta)+\cdots
\end{aligned}
$$

A straightforward algebraic manipulation allows to represent (4) without complex numbers:

$$
\begin{aligned}
& \boldsymbol{u}=2 r^{\alpha_{1 \Re}} \cos \left(\alpha_{1 \mathfrak{J}} \ln r\right)\left(A_{1 \Re}\left(x_{3}\right) \boldsymbol{\varphi}_{0 \Re}^{\left(\alpha_{1}\right)}(\theta)\right. \\
& \left.-A_{1 \mathfrak{I}}\left(x_{3}\right) \boldsymbol{\varphi}_{0 \mathfrak{J}}^{\left(\alpha_{1}\right)}(\theta)\right)-2 r^{\alpha_{1 \Re}} \sin \left(\alpha_{1 \mathfrak{J}} \ln r\right) \\
& \times\left(A_{1 \mathfrak{I}}\left(x_{3}\right) \boldsymbol{\varphi}_{0 \Re}^{\left(\alpha_{1}\right)}(\theta)+A_{1 \Re}\left(x_{3}\right) \boldsymbol{\varphi}_{0 \mathfrak{\Im}}^{\left(\alpha_{1}\right)}(\theta)\right)+2 r^{1+\alpha_{1 \Re}} \\
& \times \cos \left(\alpha_{1 \mathfrak{\Im}} \ln r\right)\left(\partial_{3} A_{1 \Re}\left(x_{3}\right) \boldsymbol{\varphi}_{1 \Re}^{\left(\alpha_{1}\right)}(\theta)\right. \\
& \left.-\partial_{3} A_{1 \mathfrak{I}}\left(x_{3}\right) \varphi_{1 \mathfrak{I}}^{\left(\alpha_{1}\right)}(\theta)\right)-2 r^{1+\alpha_{1 \Re \mathfrak{R}}} \sin \left(\alpha_{1 \mathfrak{I}} \ln r\right) \\
& \times\left(\partial_{3} A_{1 \mathfrak{I}}\left(x_{3}\right) \boldsymbol{\varphi}_{1 \mathfrak{R}}^{\left(\alpha_{1}\right)}(\theta)+\partial_{3} A_{1 \mathfrak{R}}\left(x_{3}\right) \boldsymbol{\varphi}_{1 \mathfrak{I}}^{\left(\alpha_{1}\right)}(\theta)\right) \\
& +2 r^{2+\alpha_{1 \Re}} \cos \left(\alpha_{1 \mathfrak{I}} \ln r\right)\left(\partial_{3}^{2} A_{1 \Re}\left(x_{3}\right) \boldsymbol{\varphi}_{2 \Re}^{\left(\alpha_{1}\right)}(\theta)\right. \\
& \left.-\partial_{3}^{2} A_{1 \mathfrak{I}}\left(x_{3}\right) \varphi_{2 \mathfrak{I}}^{\left(\alpha_{1}\right)}(\theta)\right)-2 r^{2+\alpha_{1 \Re}} \sin \left(\alpha_{1 \mathfrak{I}} \ln r\right) \\
& \times\left(\partial_{3}^{2} A_{1 \mathfrak{J}}\left(x_{3}\right) \boldsymbol{\varphi}_{2 \Re}^{\left(\alpha_{1}\right)}(\theta)+\partial_{3}^{2} A_{1 \Re}\left(x_{3}\right) \boldsymbol{\varphi}_{2 \mathfrak{J}}^{\left(\alpha_{1}\right)}(\theta)\right)+\cdots \\
& +A_{3}\left(x_{3}\right) r^{\alpha_{3}} \boldsymbol{\varphi}_{0}^{\left(\alpha_{3}\right)}(\theta)+\partial_{3} A_{3}\left(x_{3}\right) r^{\alpha_{3}+1} \boldsymbol{\varphi}_{1}^{\left(\alpha_{3}\right)}(\theta) \\
& +\partial_{3}^{2} A_{3}\left(x_{3}\right) r^{\alpha_{3}+2} \varphi_{2}^{\left(\alpha_{3}\right)}(\theta)+\cdots
\end{aligned}
$$




\section{Benchmark problems}

$p$-Finite-element methods have been utilized for the solution of three benchmark problems: The first is a crack at a bi-material interface between two isotropic materials, the second is a crack in a compact test specimen at an interface of two anisotropic materials, and the third is an edge at the intersection of two anisotropic materials and traction free face. These solutions, including the eigen-pairs, shadow functions and ESIFs are provided in this section.

\subsection{Benchmark problem $A$ - crack at the interface of two isotropic materials}

A bi-material interface is composed of two homogeneous materials, as shown in Fig. 1. The two materials are isotropic, both having Poisson ratio of $v=0.3$, with Young's modulus $E=10$ of the upper material (material $\# 1)$ and $E=1$ of the lower material (material \#2). This example was chosen to present a slightly simplified situation having an entire series expansion available. The first three eigen-values for this example problem are [5]:

$\alpha_{1,2}=0.5 \pm \mathrm{i} 0.07581177769, \quad \alpha_{3}=0.5$

The eigen-functions and first two shadow functions for this benchmark problem are given by (3) when using the constants $C_{k}$ provided in Tables 1 and 2. The eigen-function and the first two shadow functions associated with the first two eigen-values are presented in Fig. 2.

The eigen-pairs and shadows of example $\mathrm{A}$ are obtained numerically (see [3]) and therefore (8) represents an approximation of the exact functions. This approximation however is of very high accuracy (assured by inspecting the convergence as the polynomial degree is increased to high values).

The chosen ESIFs are polynomials of degree 2:

$A_{1,2}\left(x_{3}\right)=\left(3+4 x_{3}+5 x_{3}^{2}\right) \pm \mathrm{i}\left(2+3 x_{3}+4 x_{3}^{2}\right)$,

$A_{3}\left(x_{3}\right)=5+4 x_{3}+2 x_{3}^{2}$

therefore the solution in the vicinity of the edge is:

$$
\begin{aligned}
& \boldsymbol{u}=\left(\left(3+4 x_{3}+5 x_{3}^{2}\right)+\mathrm{i}\left(2+3 x_{3}+4 x_{3}^{2}\right)\right) r^{\alpha_{1 \Re R}+l \alpha_{1 \mathfrak{S}}}\left(\boldsymbol{\varphi}_{0 \Re}^{\left(\alpha_{1}\right)}(\theta)\right. \\
& \left.+\imath \boldsymbol{\varphi}_{0 \mathfrak{J}}^{\left(\alpha_{1}\right)}(\theta)\right)+\left(4+10 x_{3}+\imath\left(3+8 x_{3}\right)\right) r^{1+\alpha_{1 \Re}+l \alpha_{1 \mathfrak{I}}}\left(\boldsymbol{\varphi}_{1 \Re}^{\left(\alpha_{1}\right)}(\theta)\right. \\
& \left.+\imath \boldsymbol{\varphi}_{1 \mathfrak{J}}^{\left(\alpha_{1}\right)}(\theta)\right)+(10+\imath 8) r^{2+\alpha_{1 \Re}+l \alpha_{1 \mathfrak{S}}}\left(\boldsymbol{\varphi}_{2 \mathfrak{R}}^{\left(\alpha_{1}\right)}(\theta)+\imath \boldsymbol{\varphi}_{2 \mathfrak{J}}^{\left(\alpha_{1}\right)}(\theta)\right) \\
& +\left(\left(3+4 x_{3}+5 x_{3}^{2}\right)-\mathrm{i}\left(2+3 x_{3}+4 x_{3}^{2}\right)\right) r^{\alpha_{1 \Re}-l \alpha_{1 \mathfrak{N}}}\left(\boldsymbol{\varphi}_{0 \Re}^{\left(\alpha_{1}\right)}(\theta)\right. \\
& \left.-\imath \boldsymbol{\varphi}_{0 \mathfrak{I}}^{\left(\alpha_{1}\right)}(\theta)\right)+\left(4+10 x_{3}-\imath\left(3+8 x_{3}\right)\right) r^{1+\alpha_{1 \Re}-l \alpha_{1 \mathfrak{S}}}\left(\boldsymbol{\varphi}_{1 \Re}^{\left(\alpha_{1}\right)}(\theta)\right. \\
& \left.-\imath \boldsymbol{\varphi}_{1 \mathfrak{I}}^{\left(\alpha_{1}\right)}(\theta)\right)+(10-\imath 8) r^{2+\alpha_{1 \Re}-l \alpha_{1 \mathfrak{S}}}\left(\boldsymbol{\varphi}_{2 \Re}^{\left(\alpha_{1}\right)}(\theta)-\imath \boldsymbol{\varphi}_{2 \mathfrak{I}}^{\left(\alpha_{1}\right)}(\theta)\right) \\
& +\left(5+4 x_{3}+2 x_{3}^{2}\right) r^{\alpha_{3}} \boldsymbol{\varphi}_{0}^{\left(\alpha_{3}\right)}(\theta)+4\left(1+x_{3}\right) r^{\alpha_{3}+1} \boldsymbol{\varphi}_{1}^{\left(\alpha_{3}\right)}(\theta) \\
& +4 r^{\alpha_{3}+2} \varphi_{2}^{\left(\alpha_{3}\right)}(\theta)
\end{aligned}
$$

For the domain in Fig. 1 defined by $\left\{\left(r, \theta, x_{3}\right) \mid 0 \leqslant\right.$ $\left.r \leqslant 1,0 \leqslant \theta \leqslant 2 \pi,-1 \leqslant x_{3} \leqslant 1\right\}$ on which traction free boundary conditions on the crack faces and Dirichlet boundary conditions on $r=1$ and $x_{3}= \pm 1$ are prescribed according to (7),(8), one obtains the exact solution (7),(8) at each $r, \theta, x_{3}$. The crack faces are traction free.

To examine the accuracy of series (8) we computed two error measures. First, we constructed a very refined finite-

\begin{tabular}{|c|c|c|c|c|c|c|c|c|c|}
\hline \multicolumn{5}{|c|}{$0 \leqslant \theta \leqslant 180$} & \multicolumn{5}{|c|}{$180 \leqslant \theta \leqslant 360$} \\
\hline$\varphi_{0}$ & & $\varphi_{1}$ & $\varphi_{2}$ & & $\varphi_{0}$ & & $\varphi_{1}$ & $\varphi_{2}$ & \\
\hline$\varphi_{r}$ & $\varphi_{\theta}$ & $\varphi_{x_{3}}$ & $\varphi_{r}$ & $\varphi_{\theta}$ & $\varphi_{r}$ & $\varphi_{\theta}$ & $\varphi_{x_{3}}$ & $\varphi_{r}$ & $\varphi_{\theta}$ \\
\hline
\end{tabular}
element mesh of the domain in Fig. 1 and applied bound-

\begin{tabular}{|c|c|c|c|c|c|c|c|c|c|c|}
\hline & Real part & & & & & & & & & \\
\hline$C_{1}$ & 0 & 0 & 0 & 0 & 0 & -0.008296 & 0.284280 & -0.005842 & -0.028608 & -0.075810 \\
\hline$C_{2}$ & 0 & 0 & 0 & 0 & 0 & 0 & 0 & 0 & 0 & 0 \\
\hline$C_{3}$ & 0.001340 & -0.044575 & 0.001865 & -0.003996 & 0.008624 & 0 & 0 & 0 & 0 & 0 \\
\hline$C_{4}$ & -0.000091 & -0.000072 & 0.000430 & -0.001656 & 0.000036 & -0.003046 & -0.000825 & 0.001219 & 0.016147 & 0.004843 \\
\hline$C_{5}$ & 0.001398 & -0.021071 & 0.000732 & -0.001924 & 0.005854 & 0.010789 & -0.259810 & 0.004349 & 0.010160 & 0.068870 \\
\hline$C_{8}$ & -0.022086 & 0.000175 & 0.020100 & -0.005694 & 0.002366 & -0.184330 & -0.000335 & 0.128240 & -0.036658 & -0.030244 \\
\hline$C_{9}$ & -0.021644 & -0.000034 & -0.050039 & 0.016421 & -0.000913 & -0.258360 & 0.002935 & -0.415450 & 0.146510 & 0.011816 \\
\hline \multirow[t]{2}{*}{$C_{11}$} & -0.000215 & -0.000166 & 0.000803 & 0.000509 & -0.001307 & 0.003889 & -0.002810 & -0.005295 & 0.003218 & 0.016573 \\
\hline & \multicolumn{10}{|c|}{ Imaginary part } \\
\hline$C_{1}$ & 0 & 0 & 0 & 0 & 0 & -0.137680 & 0.019182 & 0.183620 & -0.034211 & -0.027624 \\
\hline$C_{2}$ & 0 & 0 & 0 & 0 & 0 & 0 & 0 & 0 & 0 & 0 \\
\hline$C_{3}$ & 0.027840 & -0.002089 & -0.013019 & -0.001744 & 0.000338 & 0 & 0 & 0 & 0 & 0 \\
\hline$C_{4}$ & -0.000381 & -0.000461 & -0.001204 & -0.000522 & -0.000182 & 0.004935 & -0.003912 & 0.010488 & 0.002167 & 0.010478 \\
\hline$C_{5}$ & 0.004731 & -0.001732 & -0.027566 & 0.004913 & 0.000644 & 0.109710 & -0.016178 & -0.208420 & 0.035949 & 0.016541 \\
\hline$C_{8}$ & 0.003406 & -0.040553 & 0.002676 & -0.003072 & 0.007558 & 0.028426 & -0.316950 & 0.027023 & -0.006660 & 0.026137 \\
\hline$C_{9}$ & -0.003448 & -0.002680 & -0.000873 & 0.001912 & -0.001576 & -0.028265 & -0.129140 & -0.008292 & 0.008246 & 0.019970 \\
\hline$C_{11}$ & 0.000039 & -0.000763 & -0.000399 & 0.000716 & -0.000460 & -0.000320 & 0.007610 & -0.004628 & -0.004693 & 0.007187 \\
\hline
\end{tabular}
ary conditions according to (8) on its boundary. After increasing the polynomial order over all elements up to $p=8$ and obtaining a FE solution with high accuracy we extracted numerically the pointwise displacements at 10

Table 1

$C_{k}$ of real part of the eigen-function, and first two shadow functions associated with $\alpha_{1,2}=0.5 \pm i 0.075812$ - example A

$\varphi_{x_{3}}$ associated with $\varphi_{0}, \varphi_{r}, \varphi_{\theta}$ associated with $\varphi_{1}$ and $\varphi_{x_{3}}$ associated with $\varphi_{2}$ are zero. The constants $C_{6}, C_{7}, C_{10}, C_{12}, C_{13}, C_{14}$ are zero. 
Table 2

$C_{k}$ of eigen-functions and first two shadow functions associated with $\alpha_{3}=0.5-$ example A

\begin{tabular}{|c|c|c|c|c|c|c|c|c|}
\hline & \multicolumn{4}{|c|}{$0 \leqslant \theta \leqslant 180$} & \multicolumn{4}{|c|}{$180 \leqslant \theta \leqslant 360$} \\
\hline & \multirow{2}{*}{$\begin{array}{c}\boldsymbol{\varphi}_{0} \\
\varphi_{x_{3}}\end{array}$} & \multicolumn{2}{|l|}{$\varphi_{1}$} & \multirow{2}{*}{$\begin{array}{l}\boldsymbol{\varphi}_{2} \\
\varphi_{x_{3}}\end{array}$} & \multirow{2}{*}{$\begin{array}{l}\varphi_{0} \\
\varphi_{x_{3}}\end{array}$} & \multicolumn{2}{|l|}{$\varphi_{1}$} & \multirow{2}{*}{$\begin{array}{l}\boldsymbol{\varphi}_{2} \\
\varphi_{x_{3}}\end{array}$} \\
\hline & & $\varphi_{r}$ & $\varphi_{\theta}$ & & & $\varphi_{r}$ & $\varphi_{\theta}$ & \\
\hline$C_{1}$ & 0 & 0 & 0 & 0 & -0.000006 & -0.000061 & -0.240940 & 0.000042 \\
\hline$C_{2}$ & 0 & 0 & 0 & 0 & 0 & 0 & 0 & 0 \\
\hline$C_{3}$ & 0 & -0.000005 & 0.024094 & 0.000001 & 0 & 0 & 0 & 0 \\
\hline$C_{4}$ & 0 & -0.000002 & 0.024096 & -0.000002 & 0 & 0.000018 & 0.240960 & -0.000023 \\
\hline$C_{5}$ & 0 & -0.000005 & -0.000002 & 0.000002 & 0.000001 & 0.000047 & -0.000017 & -0.000017 \\
\hline$C_{8}$ & 0.072294 & -0.024104 & 0.000004 & -0.012046 & 0.722940 & -0.241040 & 0.000073 & -0.120460 \\
\hline$C_{9}$ & 0 & 0.000003 & 0.000003 & -0.000002 & 0 & 0.000033 & -0.000028 & -0.000017 \\
\hline$C_{11}$ & 0 & -0.024096 & -0.000001 & 0.000802 & 0 & -0.240960 & 0.000004 & 0.008026 \\
\hline
\end{tabular}

$\varphi_{r}, \varphi_{\theta}$ associated with $\varphi_{0}, \varphi_{x_{3}}$ associated with $\varphi_{1}$ and $\varphi_{r}, \varphi_{\theta}$ associated with $\varphi_{2}$ are zero. The constants $C_{6}, C_{7}, C_{10}, C_{12}, C_{13}, C_{14}$ are zero.
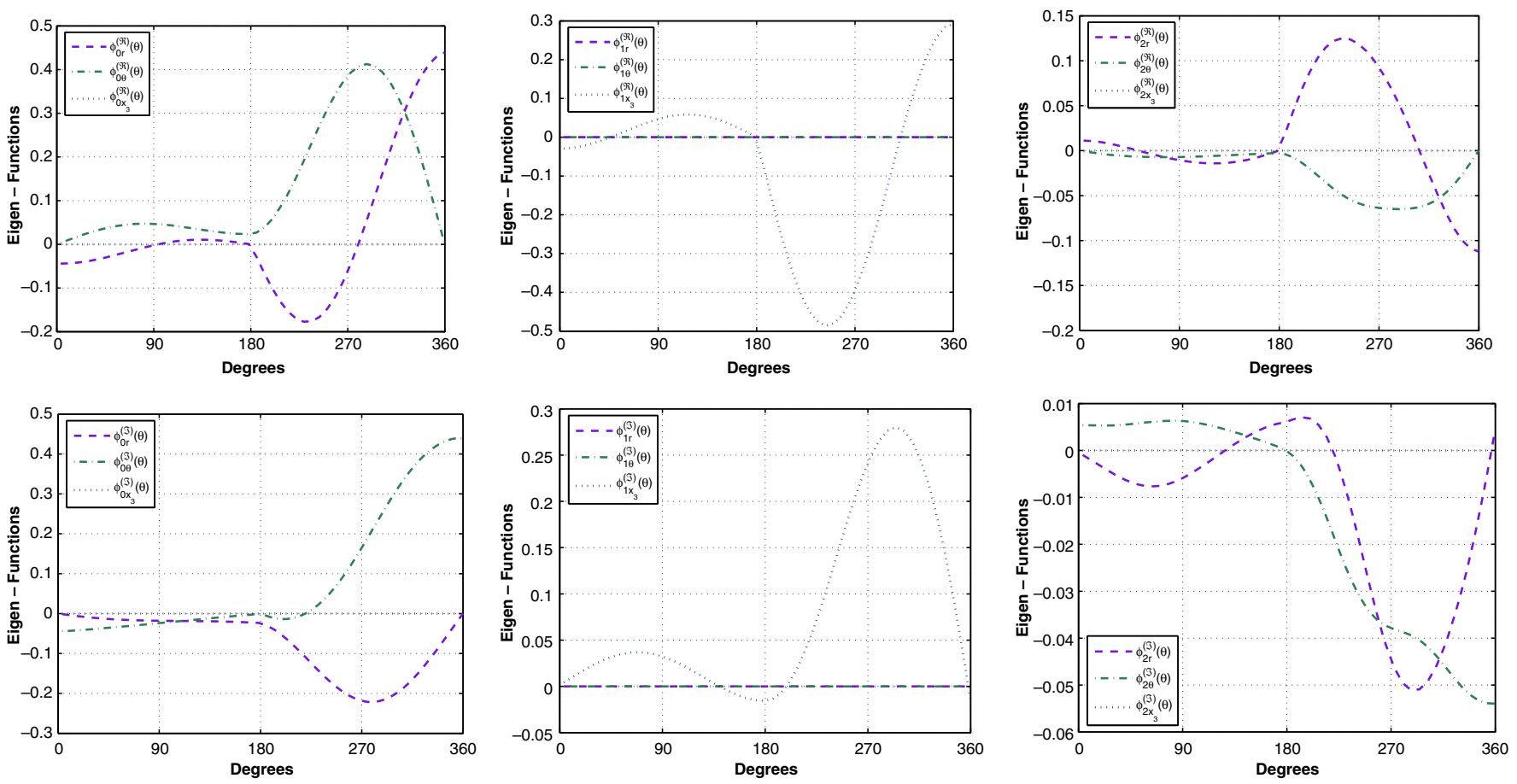

Fig. 2. The real and complex (top \& bottom, respectively) part of the eigen-functions $\varphi_{0}, \varphi_{1}, \varphi_{2}$ associated with $\alpha_{1,2}=0.5 \pm i 0.075812$ : example A.

randomly selected points in the domain. These were within less than $0.1 \%$ relative error compared to (8). Thereafter we computed the traction at the two traction free crack surfaces, using (8) (having $\alpha_{3}$ only) where $A_{3}=5+4 x_{3}+2 x_{3}^{2}$ and the $C_{k}$ coefficients in Table 2. Although the traction should be zero (traction free boundary conditions on the two surfaces) the calculated traction is of an order of $10^{-5}$ due to small numerical errors.

\subsection{Example G-CTS: crack at the interface of two anisotropic materials}

Consider the classical compact tension specimen (CTS) of a constant thickness $2\left(-1<x_{3}<1\right)$, shown in Fig. 3 . The CTS's faces are traction free and it is loaded by bear- ing loads at the tearing holes having an equivalent force of 100 in the $x_{2}$ direction as seen in Fig. 4. Although the loading is independent of $x_{3}$, because of the vertex singularities at $x_{3}= \pm 1$ the ESIFs changes as the vertices are approached.

The CTS is made of two orthotropic materials made of the same high-modulus graphite-epoxy composite with different fiber orientation. The material properties are:

$$
\begin{aligned}
& E_{L}=1.38 \times 10^{5} \mathrm{MPa} \quad E_{T}=E_{z}=1.45 \times 10^{4} \mathrm{MPa} \\
& G_{L T}=G_{L z}=G_{T z}=0.586 \times 10^{4} \mathrm{MPa} \quad v_{L T}=v_{L z}=v_{T z}=0.21
\end{aligned}
$$

The subscripts $L, T, z$ refer to fiber, transverse and thickness direction of the composite. The fibers orientation in the upper material is $+45^{\circ}$ and in the lower material $-45^{\circ}$. 

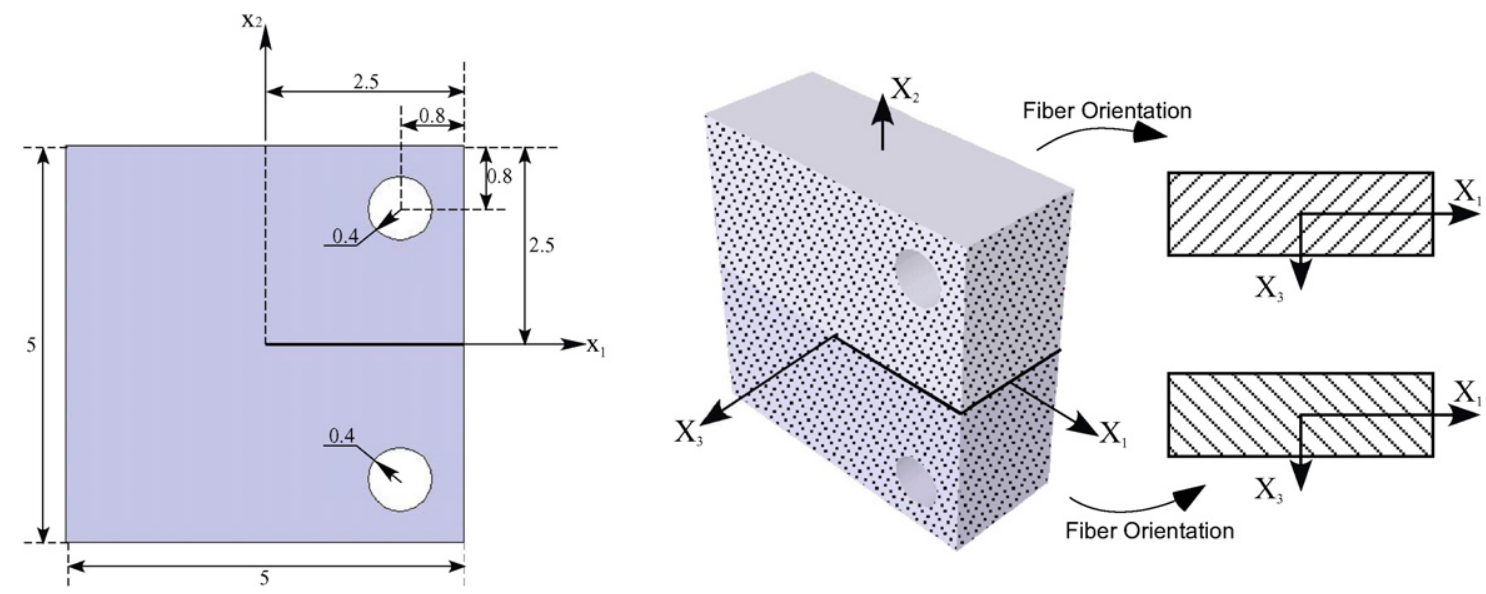

Fig. 3. Example G: Dimensions of the CTS (thickness is $2,-1<x_{3}<1$ ).

The first three eigen-values for this example problem are:

$\alpha_{1,2}=0.5 \pm \mathrm{i} 0.034328, \quad \alpha_{3}=0.5$

Unlike Example A, herein an infinite number of shadow functions in (3) exist because the ESIFs are not necessary polynomials. The first three eigen-functions of engineering importance (associated with eigen-values smaller than 1 resulting in singular stresses) and first two shadow functions (for each eigen-function) in the solution (3) are given by the $C_{k}$ 's provided in Tables 3 and 4 . The first three ESIFs were computed by the quasi-dual function method in conjunction with the p-version of the finite-element method (see FE mesh in Fig. 4) according to [3] and presented in Fig. 5.

\subsection{Example M-CTS: two cross-ply anisotropic materials}

Consider a bi-material interface structure of constant thickness $2\left(-1<x_{3}<1\right)$, shown in Fig. 6. This structure is similar to the CTS in previous section, having traction free boundary conditions on all faces except the bearing loads of a magnitude of 100 in the $x_{2}$ direction at the holes, see Fig. 7. Because of the vertex singularities at $x_{3}= \pm 1$ the first ESIF tends to infinity as the vertices are approached.

This structure is made of two orthotropic materials made of the same high-modulus graphite-epoxy system with different fiber orientation. Material properties are given in (9) with fiber orientation in the upper material at $x_{1}$ direction whereas the orientation in the lower material is at $x_{3}$ direction.

Only the first eigen-value for this example problem results in a singular stress (and is of very mild singularity):

$\alpha_{1}=0.966629$

other eigen-values are larger or equal to 1 and therefore are of no engineering importance. As for Example G, in this example there is an infinite number of shadow functions in (3) also. Only the first eigen-function and its shadows in the solution (3), are given by the coefficients $C_{k}$ 's in Table 5 .

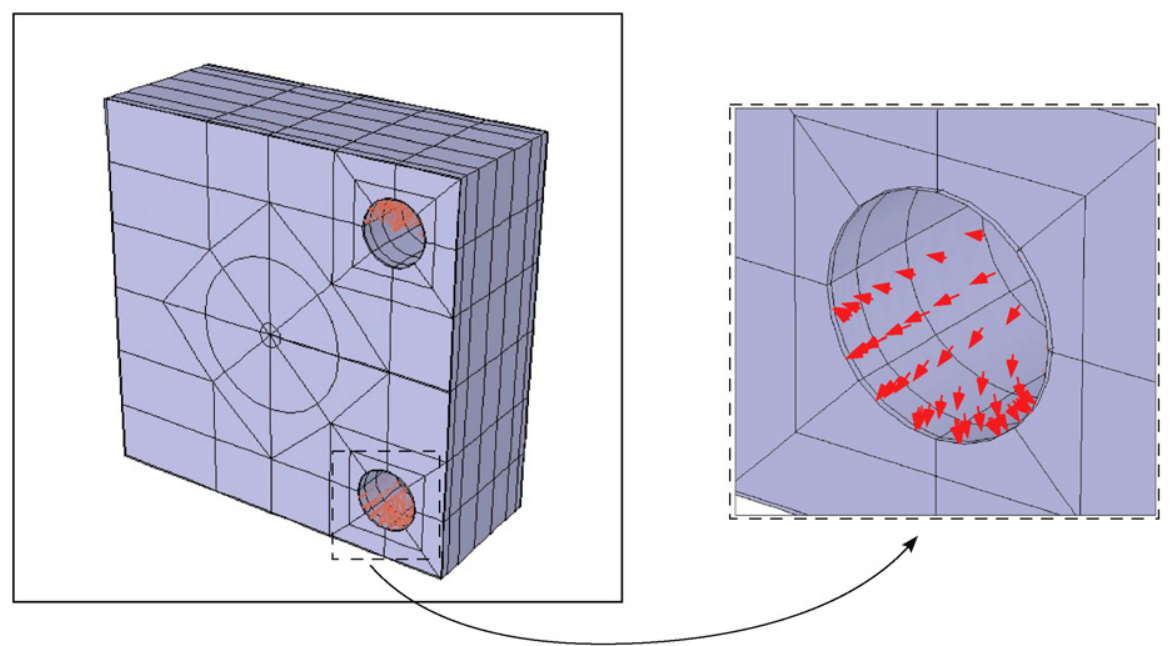

Fig. 4. Example G: The $p$-finite-element mesh used for ESIF extraction. 
$C_{k}$ of real and imaginary parts of eigen-function and first two shadow functions associated with $\alpha_{1,2}=0.5 \pm 10.034328$ - example $\mathrm{G}$

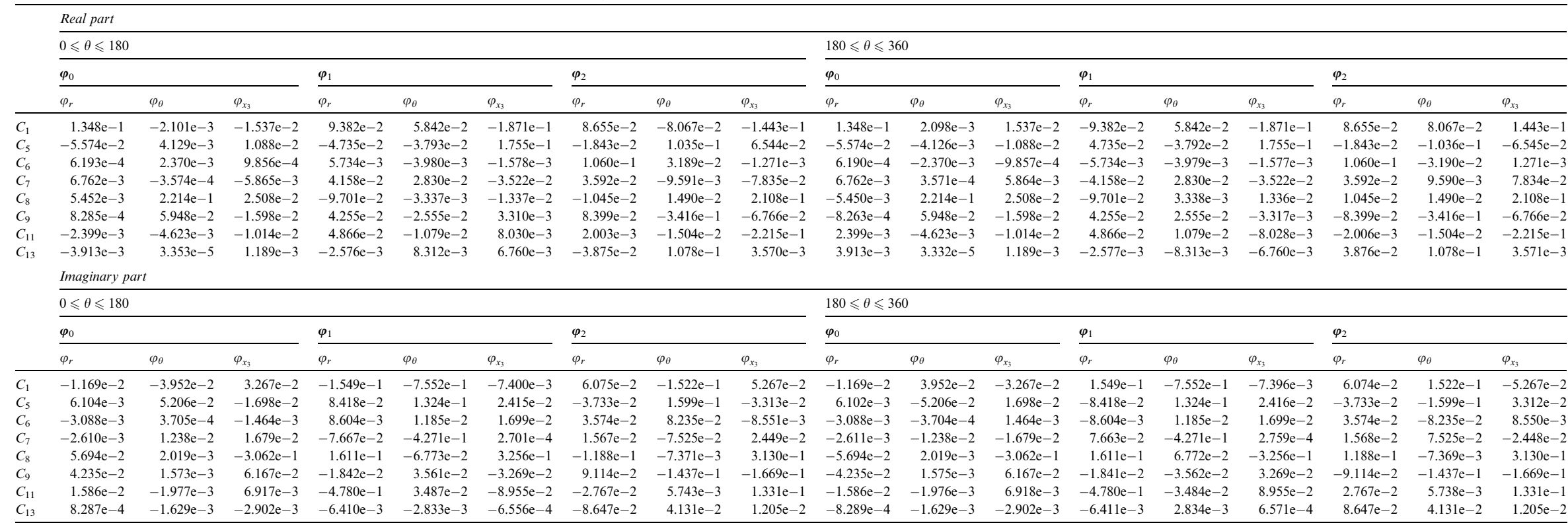

Table 4

$C_{k}$ of eigen-function and first two shadow functions associated with $\alpha_{3}=0.5-$ example $\mathrm{G}$

\begin{tabular}{|c|c|c|c|c|c|c|c|c|c|c|c|c|c|c|c|c|c|c|}
\hline & \multicolumn{9}{|l|}{$0 \leqslant \theta \leqslant 180$} & \multicolumn{9}{|c|}{$180 \leqslant \theta \leqslant 360$} \\
\hline & \multicolumn{3}{|l|}{$\varphi_{0}$} & \multicolumn{3}{|l|}{$\varphi_{1}$} & \multicolumn{3}{|l|}{$\varphi_{2}$} & \multicolumn{3}{|l|}{$\varphi_{0}$} & \multicolumn{3}{|l|}{$\underline{\varphi_{1}}$} & \multicolumn{3}{|l|}{$\underline{\varphi_{2}}$} \\
\hline & $\varphi_{r}$ & $\varphi_{\theta}$ & $\varphi_{x_{3}}$ & $\varphi_{r}$ & $\varphi_{\theta}$ & $\varphi_{x_{3}}$ & $\varphi_{r}$ & $\varphi_{\theta}$ & $\varphi_{x_{3}}$ & $\varphi_{r}$ & $\varphi_{\theta}$ & $\varphi_{x_{3}}$ & $\varphi_{r}$ & $\varphi_{\theta}$ & $\varphi_{x_{3}}$ & $\varphi_{r}$ & $\varphi_{\theta}$ & $\varphi_{x_{3}}$ \\
\hline$C_{1}$ & $-8.481 \mathrm{e}-3$ & $2.835 \mathrm{e}-1$ & $5.307 \mathrm{e}-2$ & $-1.600 \mathrm{e}-2$ & $5.635 e-2$ & $1.406 \mathrm{e}-2$ & $-4.022 \mathrm{e}-2$ & $2.631 \mathrm{e}-2$ & $-2.032 \mathrm{e}-2$ & $8.483 \mathrm{e}-3$ & $2.835 \mathrm{e}-1$ & $5.307 \mathrm{e}-2$ & $-1.600 e-2$ & $-5.635 \mathrm{e}-2$ & $-1.406 \mathrm{e}-2$ & $4.021 \mathrm{e}-2$ & $2.631 \mathrm{e}-2$ & $-2.032 \mathrm{e}-2$ \\
\hline$C_{5}$ & $-2.947 e-3$ & $-1.511 \mathrm{e}-1$ & $-2.749 \mathrm{e}-2$ & $7.425 \mathrm{e}-3$ & $-8.398 \mathrm{e}-2$ & $-9.236 \mathrm{e}-3$ & $4.666 \mathrm{e}-2$ & $4.702 \mathrm{e}-2$ & $1.171 \mathrm{e}-2$ & $2.941 \mathrm{e}-3$ & $-1.511 \mathrm{e}-1$ & $-2.749 \mathrm{e}-2$ & $7.429 \mathrm{e}-3$ & $8.398 \mathrm{e}-2$ & $9.260 \mathrm{e}-3$ & $-4.666 \mathrm{e}-2$ & $4.702 \mathrm{e}-2$ & $1.171 \mathrm{e}-2$ \\
\hline$C_{6}$ & $-7.449 \mathrm{e}-3$ & $1.231 \mathrm{e}-4$ & $-2.476 \mathrm{e}-3$ & $5.865 e-4$ & $-2.151 e-3$ & $5.374 \mathrm{e}-4$ & $1.550 \mathrm{e}-2$ & $8.627 \mathrm{e}-2$ & $1.169 \mathrm{e}-3$ & $7.448 \mathrm{e}-3$ & $1.227 \mathrm{e}-4$ & $-2.476 \mathrm{e}-3$ & $5.869 \mathrm{e}-4$ & $2.151 \mathrm{e}-3$ & $-5.373 \mathrm{e}-4$ & $-1.549 \mathrm{e}-2$ & $8.628 \mathrm{e}-2$ & $1.168 \mathrm{e}-3$ \\
\hline$C_{7}$ & $-4.162 \mathrm{e}-3$ & $1.253 \mathrm{e}-2$ & $2.747 \mathrm{e}-2$ & $-9.211 \mathrm{e}-3$ & $-2.545 \mathrm{e}-2$ & $4.509 \mathrm{e}-3$ & $-9.553 \mathrm{e}-3$ & $1.597 \mathrm{e}-2$ & $-9.236 \mathrm{e}-3$ & $4.163 \mathrm{e}-3$ & $1.253 \mathrm{e}-2$ & $2.747 e-2$ & $-9.209 \mathrm{e}-3$ & $2.545 \mathrm{e}-2$ & $-4.509 \mathrm{e}-3$ & $9.553 \mathrm{e}-3$ & $1.598 \mathrm{e}-2$ & $-9.241 \mathrm{e}-3$ \\
\hline$C_{8}$ & $-1.667 \mathrm{e}-1$ & $-1.480 \mathrm{e}-3$ & $7.436 \mathrm{e}-2$ & $-7.950 \mathrm{e}-2$ & $-3.087 \mathrm{e}-5$ & $-1.286 e-1$ & $6.227 e-2$ & $3.843 \mathrm{e}-2$ & $-1.380 e-1$ & $-1.667 \mathrm{e}-1$ & $1.483 \mathrm{e}-3$ & $-7.435 e-2$ & $7.950 \mathrm{e}-2$ & $-3.161 \mathrm{e}-5$ & $-1.286 \mathrm{e}-1$ & $6.227 \mathrm{e}-2$ & $-3.843 \mathrm{e}-2$ & $1.380 \mathrm{e}-1$ \\
\hline$C_{9}$ & $-1.588 \mathrm{e}-1$ & $5.709 \mathrm{e}-3$ & $6.206 \mathrm{e}-2$ & $-8.422 \mathrm{e}-2$ & $-3.830 \mathrm{e}-4$ & $6.182 \mathrm{e}-1$ & $-1.790 e-1$ & $-4.282 \mathrm{e}-2$ & $8.877 \mathrm{e}-2$ & $-1.588 \mathrm{e}-1$ & $-5.703 e-3$ & $-6.206 \mathrm{e}-2$ & $8.422 \mathrm{e}-2$ & $-3.863 \mathrm{e}-4$ & $6.182 \mathrm{e}-1$ & $-1.790 \mathrm{e}-1$ & 4.282e- -2 & $-8.877 \mathrm{e}-2$ \\
\hline$C_{11}$ & $1.997 \mathrm{e}-2$ & $-2.793 e-4$ & $1.130 \mathrm{e}-2$ & $-2.807 \mathrm{e}-2$ & $3.932 \mathrm{e}-4$ & $4.124 \mathrm{e}-3$ & $1.505 \mathrm{e}-3$ & $-9.447 e-3$ & $7.642 \mathrm{e}-2$ & $1.997 \mathrm{e}-2$ & $2.785 e-4$ & $-1.130 \mathrm{e}-2$ & $2.807 \mathrm{e}-2$ & $3.915 \mathrm{e}-4$ & $4.125 \mathrm{e}-3$ & $1.504 \mathrm{e}-3$ & $9.445 \mathrm{e}-3$ & $-7.642 \mathrm{e}-2$ \\
\hline$C_{13}$ & $1.138 \mathrm{e}-3$ & $-3.937 \mathrm{e}-3$ & $-3.822 \mathrm{e}-3$ & $4.263 \mathrm{e}-3$ & $7.969 \mathrm{e}-5$ & $-1.105 e-3$ & $-8.213 \mathrm{e}-2$ & $1.366 \mathrm{e}-2$ & $-5.840 \mathrm{e}-3$ & $1.138 \mathrm{e}-3$ & $3.937 \mathrm{e}-3$ & $3.822 \mathrm{e}-3$ & $-4.263 \mathrm{e}-3$ & $7.989 \mathrm{e}-5$ & $-1.105 e-3$ & $-8.213 \mathrm{e}-2$ & $-1.365 \mathrm{e}-2$ & $5.840 \mathrm{e}-3$ \\
\hline
\end{tabular}

The constants $C_{2}, C_{3}, C_{4}, C_{10}, C_{12}, C_{14}$ are zero. 

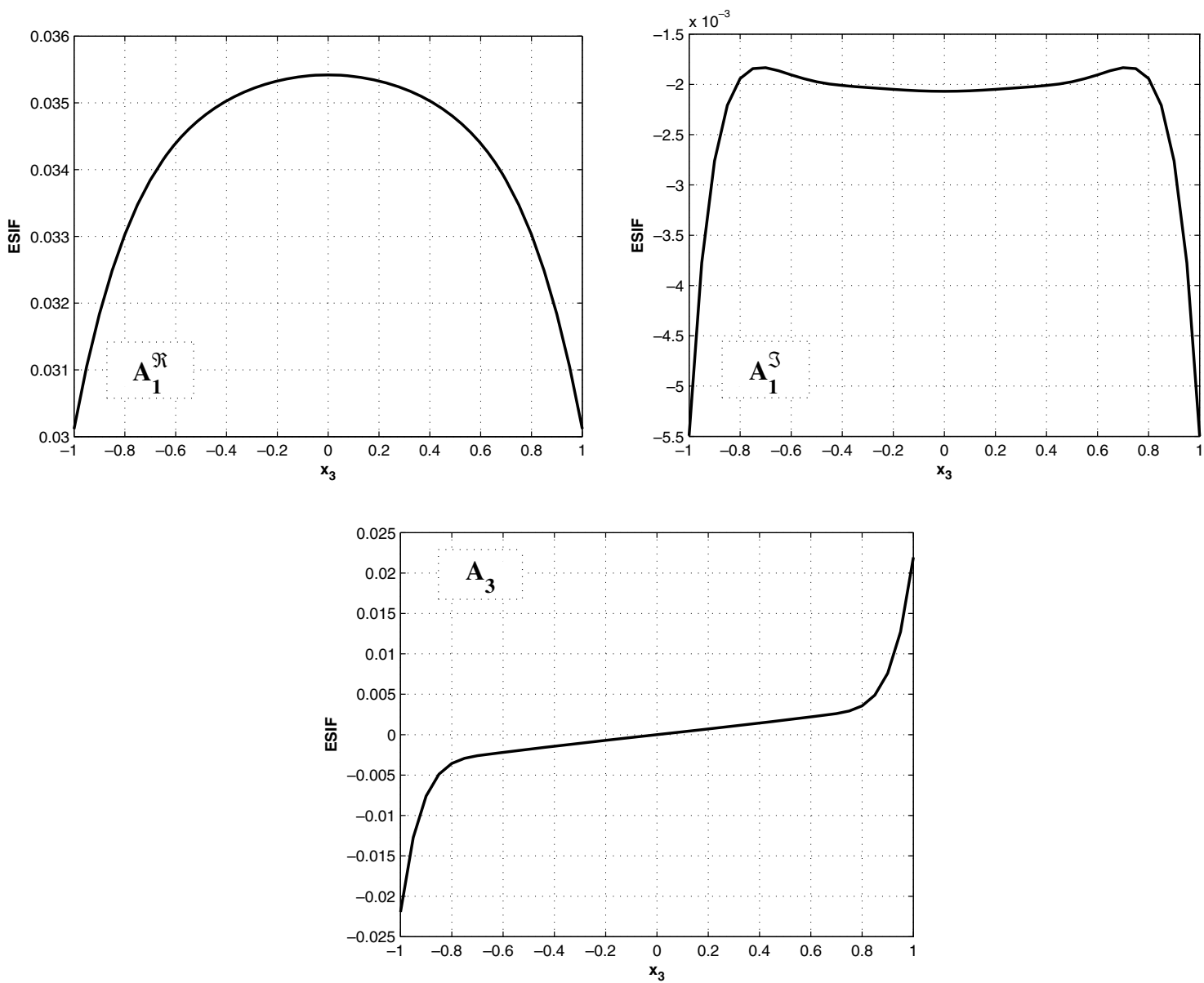

Fig. 5. ESIFs for example G: $A_{1}^{\Re}\left(x_{3}\right) \pm \imath A_{1}^{\widetilde{\Im}}\left(x_{3}\right)$ and $A_{3}\left(x_{3}\right)$.
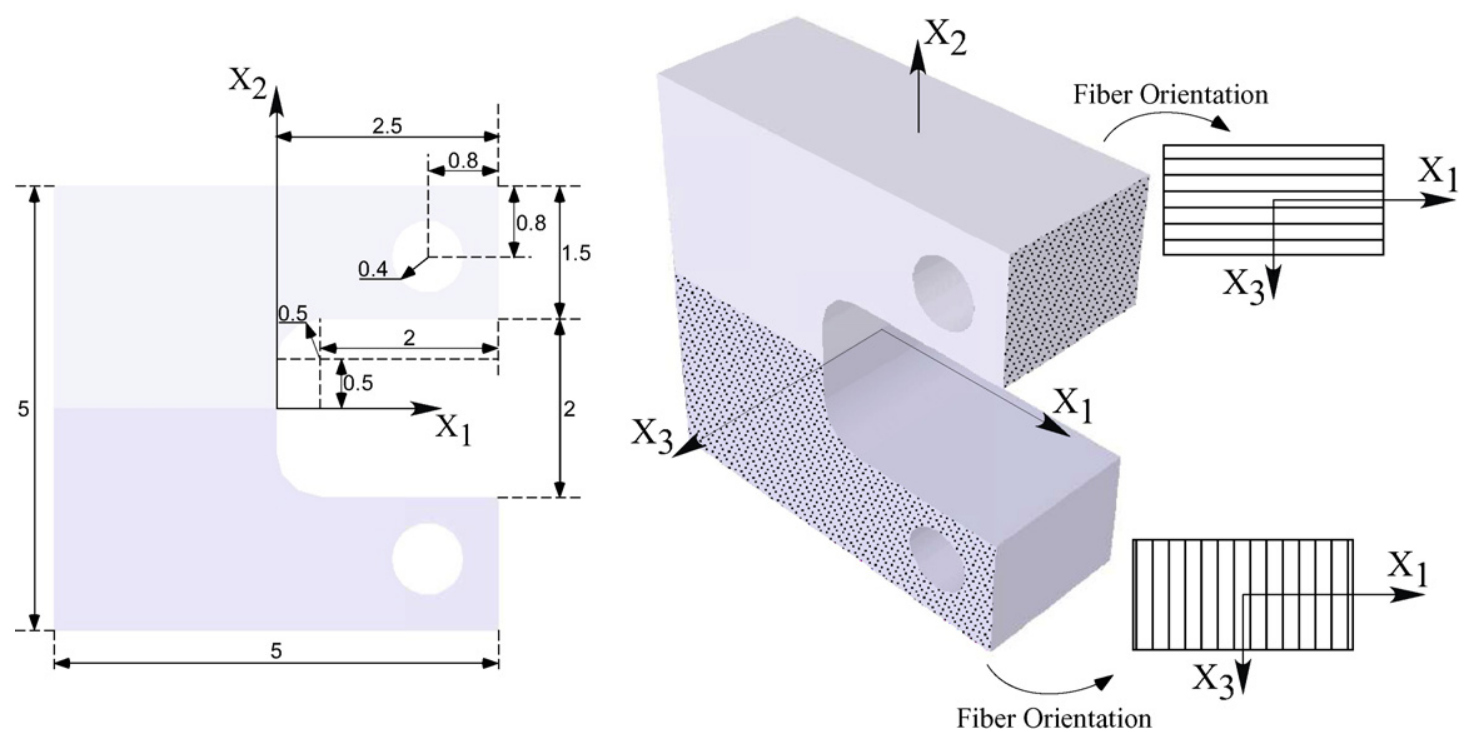

Fig. 6. Example M: Dimensions (thickness is $2,-1<x_{3}<1$ ).

The first ESIF (the only one of engineering relevance) is extracted and plotted in Fig. 8. The influence of the vertex singularities at $x_{3}= \pm 1$ is visible, resulting in strong gradients in ESIF (which tends to $\infty$ at the vertices).

\section{Summary}

The elastic solution in the vicinity of edges in anisotropic multi-material interfaces representative of composite mate- 


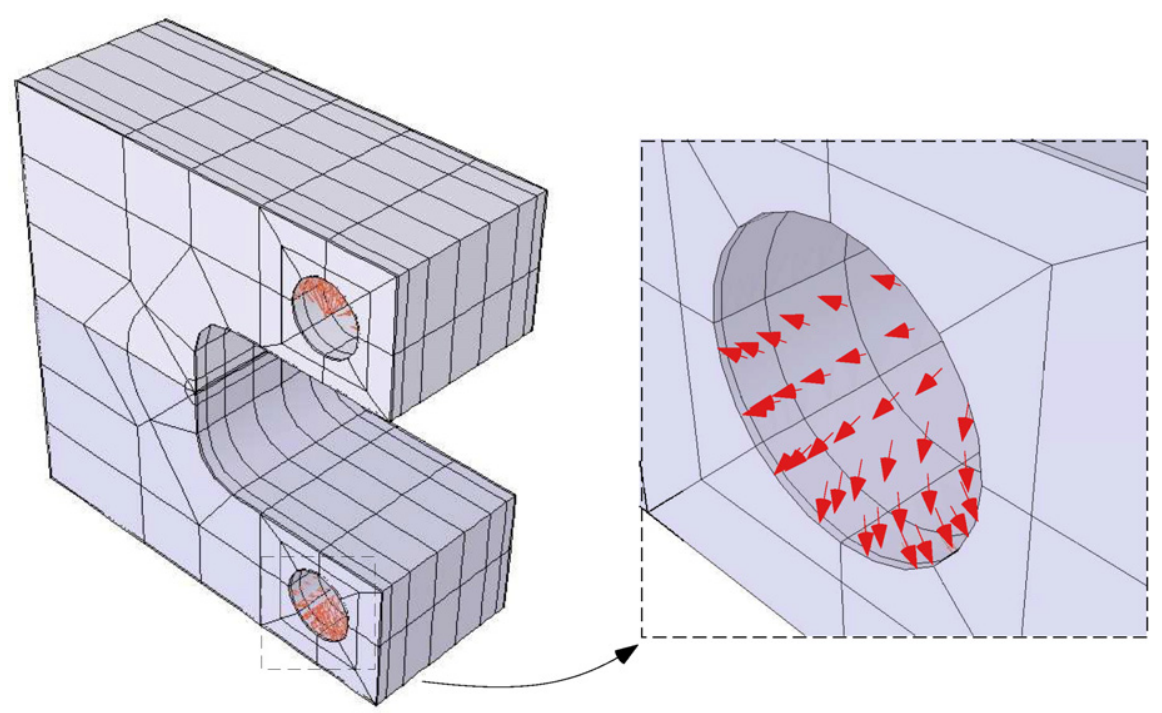

Fig. 7. Example M: The $p$-finite-element mesh used for ESIF extraction.

Table 5

$C_{k}$ of first eigen-function and first two shadow functions associated with $\alpha_{1}=0.966629$ - example M

\begin{tabular}{|c|c|c|c|c|c|c|c|c|c|c|}
\hline & \multicolumn{5}{|l|}{$0 \leqslant \theta \leqslant 180$} & \multicolumn{5}{|c|}{$180 \leqslant \theta \leqslant 360$} \\
\hline & \multicolumn{2}{|l|}{$\varphi_{0}$} & \multirow{2}{*}{$\begin{array}{l}\boldsymbol{\varphi}_{1} \\
\varphi_{x_{3}}\end{array}$} & \multicolumn{2}{|l|}{$\varphi_{2}$} & \multicolumn{2}{|l|}{$\varphi_{0}$} & \multirow{2}{*}{$\begin{array}{l}\varphi_{1} \\
\varphi_{x_{3}}\end{array}$} & \multicolumn{2}{|l|}{$\varphi_{2}$} \\
\hline & $\varphi_{r}$ & $\varphi_{\theta}$ & & $\varphi_{r}$ & $\varphi_{\theta}$ & $\varphi_{r}$ & $\varphi_{\theta}$ & & $\varphi_{r}$ & $\varphi_{\theta}$ \\
\hline$C_{1}$ & -0.057525 & 0.009735 & 0.006953 & -2.467100 & -0.328100 & 0.002383 & -0.000050 & -0.000347 & 1.810700 & -0.638130 \\
\hline$C_{2}$ & 0.008685 & 0.016553 & -0.167350 & 0.075265 & 0.240510 & 0.009705 & 0.058856 & -0.168960 & -0.006105 & -0.216040 \\
\hline$C_{3}$ & -1.434800 & 0.705620 & 0.244180 & -67.207000 & 5.058700 & -0.194930 & -0.147380 & 0.113370 & -56.949000 & 4.699900 \\
\hline$C_{8}$ & 0.039866 & -0.035057 & -0.003761 & 2.227400 & -0.720650 & 0.002422 & 0.000958 & -0.000354 & 2.168900 & 0.243620 \\
\hline$C_{9}$ & -0.023477 & 0.010291 & -0.026977 & -0.520830 & 0.101050 & -0.058518 & 0.009767 & 0.009697 & 0.449220 & -0.004979 \\
\hline$C_{10}$ & -0.031351 & 0.355330 & 0.007513 & -1.706100 & 0.619140 & -0.059358 & 0.315730 & 0.044530 & -4.468700 & 0.777580 \\
\hline
\end{tabular}

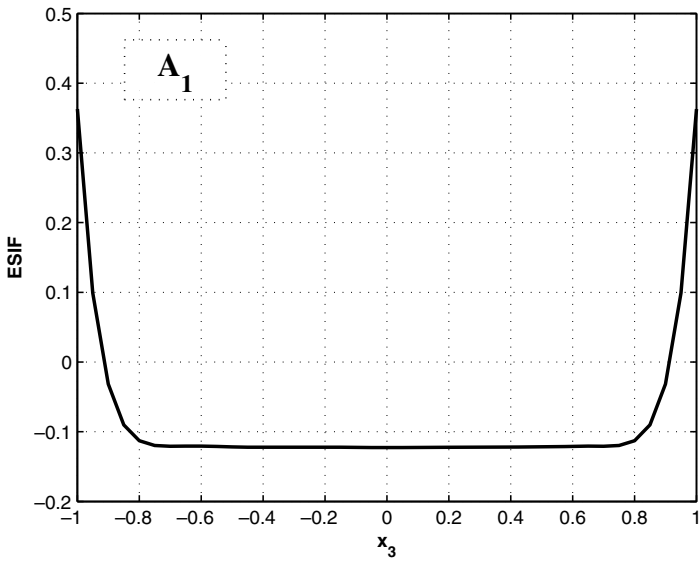

Fig. 8. Example M: First ESIF.

rials was provided for three different problems. These solutions, although being computed by numerical methods presented in [3] are of very high accuracy, therefore suggested as benchmarks for the verification of various methods aimed at computing edge stress intensity functions in composite-like structures.

\section{Acknowledgement}

First two authors gratefully acknowledge the partial support of this work by the Israel Science Foundation (Grant No. 750/07).

\section{References}

[1] Costabel M, Dauge M, Yosibash Z. A quasidual function method for extracting edge stress intensity functions. SIAM J Math Anal 2004;35(5):1177-202.

[2] Freed Y, Banks-Sills L. A through interface crack between a $\pm 45^{\circ}$ transversely isotropic pair of materials. Int J Fracture 2005(133): $1-41$.

[3] Omer N, Yosibash Z. Edge singularities in 3-D elastic anisotropic and multi-material domains. Comput Meth Appl Mech Eng, [In press].

[4] Suo Z. Singularities, interfaces and cracks in dissimilar anisotropic media. Proc Royal Soc London 1990;A427:331-58.

[5] Yosibash Z. Computing edge singularities in elastic anisotropic threedimensional domains. Int J Fract 1997;86(3):221-45.

[6] Yosibash Z. On solutions of two-dimensional linear elastostatic and heat-transfer problems in the vicinity of singular points. Int J Solids Struct 1997;34(2):243-74. 
[7] Yosibash Z, Omer N. Numerical methods for extracting edge stress intensity functions in anisotropic three-dimensional domains. Comput Meth Appl Mech Eng 2007;196:3624-49.
[8] Yosibash Z, Omer N, Costabel M, Dauge M. Edge stress intensity functions in polyhedral domains and their extraction by a quasidual function method. Int J Fract 2005;136:37-73. 